\title{
Analytical Model of a Weighted Round Robin Service System
}

\author{
Vladimir Hottmar and Bohumil Adamec \\ Department of Telecommunications and Multimedia, Faculty of Electrical Engineering, University of Žilina, 01026 Žilina, Slovakia \\ Correspondence should be addressed to Bohumil Adamec, bohumil.adamec@fel.uniza.sk
}

Received 19 September 2011; Revised 13 January 2012; Accepted 27 January 2012

Academic Editor: Yaohui Jin

Copyright ( 2012 V. Hottmar and B. Adamec. This is an open access article distributed under the Creative Commons Attribution License, which permits unrestricted use, distribution, and reproduction in any medium, provided the original work is properly cited.

\begin{abstract}
This paper presents a mathematical description of Weighted Round Robin service strategy, on which basis all the perspective QoS tools that serve to manage congestion in converged packet networks work. On the basis of the presented mathematical model, it is possible to suitably configure the operational parameters (maximum queue length, distribution of the available transfer capacity) of these tools according to required values of packet delays. The implementation of the analytical model is demonstrated on a real network segment using advanced network data traffic emulator.
\end{abstract}

\section{Introduction}

In converged IP networks, it is necessary to provide, apart from the conventional data operation, also the video and voice communication support. Because IP packet architecture was not originally intended for real-time transfers, from the point of view of interactive communication it is necessary to solve the problem of quality guarantee of multimedia services-Quality of Service (QoS). Suitably implemented QoS tools ought to guarantee a certain quality level of processing of time-sensitive interactive applications at the expense of the quality of processing of other applications that do not necessarily require real-time transfers. Without properly implemented QoS tools, the applications that do not require real-time processing might use up the available transfer capacity and in this way make impossible the transfer of time-sensitive interactive applications. The main problem of IP packet network is thus the appropriate support of multimedia communication taking place in real time and the connected suitable management of potentially occurring congestion. The appropriate implementation of QoS tools mainly requires correct setting of the operation parameters of these tools. In order to provide high Quality-of-Service (QoS) in today's high-speed converged networks, WRR mechanism assigns different priorities to different queues. WRR also ensures fair selection interval among all active queues with minimal delay and jitter [1]. In this scheduling algorithm, a weighting coefficient for each queue determines how many bytes of data the system delivers from the queue before it moves on to the next queue. The WRR mechanism cycles through the queues. For each queue, packets are sent until the number of bytes transmitted exceeds the bandwidth determined by the queue's weighting coefficient, or the queue is empty $[2,3]$. Then the WRR mechanism moves to the next queue. If a queue is empty, WRR mechanism will send packets from the next queue that has packets ready to send. This mechanism guarantees a minimum bandwidth to each queue, and allows the minimum to be exceeded if one or more of the port's other queues are idle. However, when all the queues are located, each is limited to its maximum bandwidth according to its assigned weightno queue achieves more than a predetermined portion of transfer capacity when the transmission line is under stress [4]. WRR includes several significant benefits. This scheduling mechanism can be implemented in hardware, so it can be applied to high-speed interfaces in both the core and at the edges of the network. WRR mechanism also ensures that all service classes have access to at least some configured amount of network capacity to avoid bandwidth starvation. WRR queuing provides coarse control over the percentage of output port bandwidth allocated to each service class. Classification of traffic by service class provides more equitable management and more stability for network applications than the use of priorities or preferences. WRR 


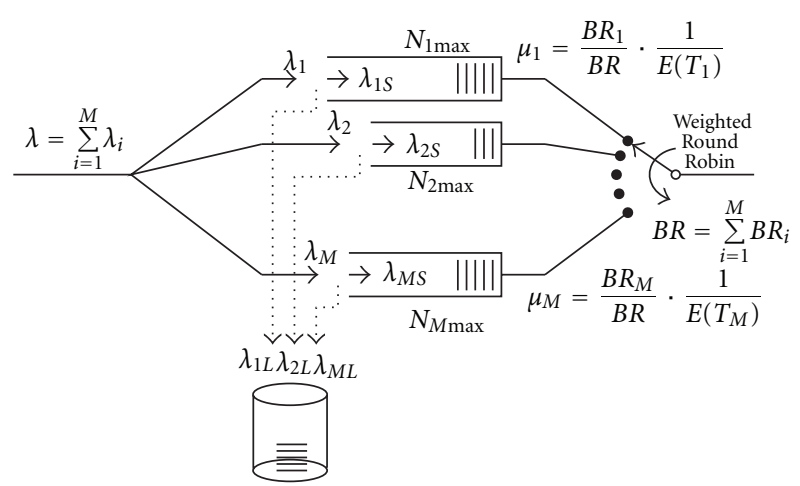

FIgURE 1: Model of Weighted Round Robin network node.

queuing is based on the belief that resource reduction is a better mechanism to control congestion than resource denial $[1,2]$. Other information about WRR models and detailed description of the status quo can be found in source [4].

\section{Analytical Modeling of Weighted Round Robin Service Strategy}

In Figure 1 we can see the model of a Weighted Round Robin network node with $M$ queues. The offered traffic with $\lambda$ intensity consists of data flows of individual queues with intensities $\lambda_{1}, \lambda_{2}$ to $\lambda_{M}$, from which a certain part $\lambda_{1 S}, \lambda_{2 S}$ to $\lambda_{M S}$ is processed by the node and a certain part $\lambda_{1 L}, \lambda_{2 L}$ to $\lambda_{M L}$ is discarded by the node. In the ith queue maximally $N_{i \max }$ packets may be stored. The average time needed to service a packet of $i$ th queue is $E\left(T_{i}\right)$. Intensity of the service of the $i$ th queue is $\mu_{i}$. The overall transfer capacity $B R$ of output interface is divided according to configuration among individual queues [4].

Proposed analytical model describes the specific conditions when the WRR service system is congested. This service mode is analyzed in order to provision a certain parameter reserve for normal noncongested mode. In the case of congestion all the queues work with the predefined portion of time period. Therefore, obtained results include a certain useful reserve for the normal noncongested mode.

Processing the traffic by the Weighted Round Robin service strategy means that the service of individual queues is realized only during certain, in general different, time intervals $\Delta_{i}$. The probability that the Weighted Round Robin network node is in the state of service of $k$ th queue is given by the relation $[4]$ :

$$
\begin{aligned}
P(i=k) & =\frac{\Delta_{k}}{\Delta_{1}+\Delta_{2}+\cdots+\Delta_{M}} \\
& =\frac{\Delta_{k}}{\Delta}=\frac{B R_{k}}{B R} .
\end{aligned}
$$

When $W_{k}^{n}$ is the average waiting time of a packet belonging to the $k$ th queue, having entered the network node during service of the $n$th queue, then the average waiting time of

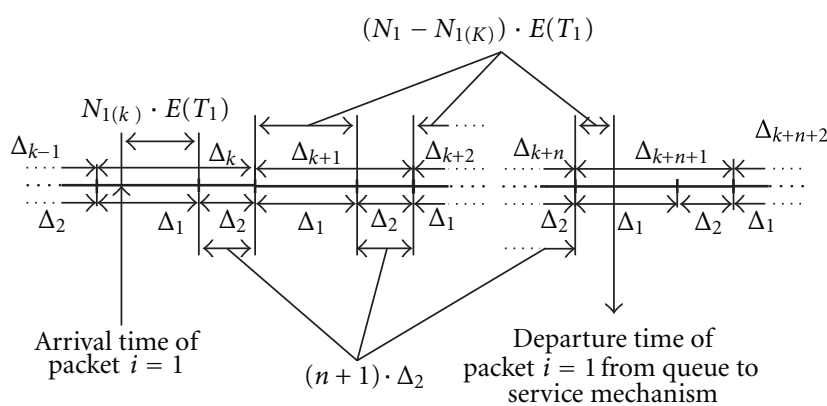

FIgURE 2: Time conditions at the arrival of $i=1$ packet during servicing the $i=1$ queue.

packet belonging to the $k$ th queue will generally be given by the formula:

$$
W_{k}=W_{k}^{1} \cdot P(i=1)+\cdots+W_{k}^{M} \cdot P(i=M) .
$$

In the case of two queues, the average waiting time of packets in the first queue is given by the relation:

$$
W_{1}=W_{1}^{1} \cdot \frac{B R_{1}}{B R}+W_{1}^{2} \cdot \frac{B R_{2}}{B R} .
$$

Figure 2 demonstrates a situation when a packet belonging to the first queue comes in the time of service of the first queue. In the moment of arrival of packet belonging to queue $i=$ 1 , on average there are $N_{1}$ packets belonging to queue $i=1$ that precedes it. The average number of packets transferred since the moment of arrival of the packet in question to the moment of finishing the interval $\Delta_{1}$ of period $\Delta_{k}$ is $N_{1(k)}$. Up to the moment of servicing the considered packet the entire interval $\Delta_{1}$ will take place $n$ times on the whole and the entire interval $\Delta_{2}(n+1)$ times altogether. Then the average waiting time of the packet, in question is given by the relation:

$$
W_{1}^{1}=N_{1(k)} \cdot E\left(T_{1}\right)+\left[N_{1}-N_{1(k)}\right] \cdot E\left(T_{1}\right)+[n+1] \cdot \Delta_{2} .
$$

The average waiting time computation comprises of the three-parts residual service time of the packet in service at the arrival, service time of the class-1 packets in queue, and the total intervals assigned to class- 2 packets. As shown in Figure 2, residual service time is included in $N_{1(k)}$ variable. In general, this variable assumes the decimal numbers; decimal part of this number represents the residual service time of the packet in service at the moment of arrival.

Since the considered packet could come during the interval $\Delta_{1}$ of period $\Delta_{k}$ at any moment with the same probability, on average it will come in the moment $\Delta_{1} / 2$ and during the period $\Delta_{k}$ it will spend time $\Delta_{1} / 2$, in the queue. During this time on average $N_{1(k)}$ packets will be processed from the arrival time of the given packet to the moment of finishing interval $\Delta_{1}$ of period $\Delta_{k}$. It is valid that

$$
N_{1(k)}=\frac{\Delta_{1}}{\left[2 \cdot E\left(T_{1}\right)\right]} .
$$

After the passage of period $\Delta_{k}$, there will be on average $N_{1}-N_{1(k)}$ packets of category $i=1$ queued before the considered packet. A certain part of these packets will be 
processed during $n$ whole intervals $\Delta_{1}$ of periods $\Delta_{k+1}$ to $\Delta_{k+n}$ and a certain residual part will be processed during interval $\Delta_{1}$ of periods $\Delta_{k+n+1}$ together with the considered packet. Since the moment of finishing period $\Delta_{k}$ to the moment of transfer of the considered packet, interval $\Delta_{1}$ will take place $n+\varepsilon$ times on whole. Variable $\varepsilon$ is the mean value of the proportion of the interval $\Delta_{1}$ of period $\Delta_{k+n+1}$ that is necessary for processing the residual part of packets. It can be expressed by

$$
\begin{aligned}
n+\varepsilon & =\frac{\left[N_{1}-N_{1(k)}\right]}{\left[\Delta_{1} / E\left(T_{1}\right)\right]} \\
& =\frac{\left[N_{1} \cdot E\left(T_{1}\right)\right]}{\Delta_{1}}-\frac{1}{2} .
\end{aligned}
$$

During one interval $\Delta_{1}$ up to $\Delta_{1} / E\left(T_{1}\right)$ packets of category $i=1$ are serviced. Thus, during the interval $\Delta_{1}$ of period $\Delta_{k+n+1}$, before the considered packet there might be up to $\left[\Delta_{1} / E\left(T_{1}\right)\right]-1$ packets serviced and on average $(1 / 2)$. $\left[\Delta_{1} / E\left(T_{1}\right)\right]-1 / 2$ packets are serviced. Since $E\left(T_{1}\right) / \Delta_{1}$ is the proportion of interval $\Delta_{1}$ needed to transfer one packet, then for the mean value of the proportion of interval $\Delta_{1}$ of period $\Delta_{k+n+1}$, needed to transfer the residual part of packets, following relation is valid:

$$
\begin{aligned}
\varepsilon & =\left[\frac{E\left(T_{1}\right)}{\left(2 \cdot \Delta_{1}\right)}\right] \cdot\left[\frac{\Delta_{1}}{E\left(T_{1}\right)-1}\right] \\
& =\left[\frac{1-E\left(T_{1}\right)}{\Delta_{1}}\right] \cdot \frac{1}{2} .
\end{aligned}
$$

It then follows that for the number of whole intervals $\Delta_{1}$ taking place since the arrival moment of a considered packet to the starting moment of its servicing, it is valid that

$$
n=\left[\frac{E\left(T_{1}\right)}{\Delta_{1}}\right] \cdot\left[N_{1}+\frac{1}{2}\right]-1 .
$$

After substituting (5) and (8) to (4), the result is

$$
W_{1}^{1}=N_{1}\left[1+\frac{\Delta_{2}}{\Delta_{1}}\right] E\left(T_{1}\right)+\frac{\Delta_{2} E\left(T_{1}\right)}{2 \Delta_{1}} .
$$

A situation when a packet belonging to the first queue is coming in the moment of servicing the second queue which can be analyzed in the same way as before. At the arrival moment of packet $i=1$, on average there are other $N_{1}$ packets in the $i=1$ queue before it. The average number of packets processed since the arrival moment of the considered packet to the moment of expiration of period $\Delta_{k}$ is $N_{2(k)}$. Until the moment of servicing the considered packet, the entire interval $\Delta_{1}$ will take place $n$ times and the entire interval $\Delta_{2} n$ times as well. It follows that the average waiting time of the considered packet is given by the relation:

$$
W_{1}^{2}=N_{2(k)} \cdot E\left(T_{2}\right)+N_{1} \cdot E\left(T_{1}\right)+n \cdot \Delta_{2} .
$$

Using the same method as before we obtain

$$
W_{1}^{2}=N_{1}\left[1+\frac{\Delta_{2}}{\Delta_{1}}\right] E\left(T_{1}\right)+\frac{\Delta_{2} E\left(T_{1}\right)}{2 \Delta_{1}}=W_{1}^{1} .
$$

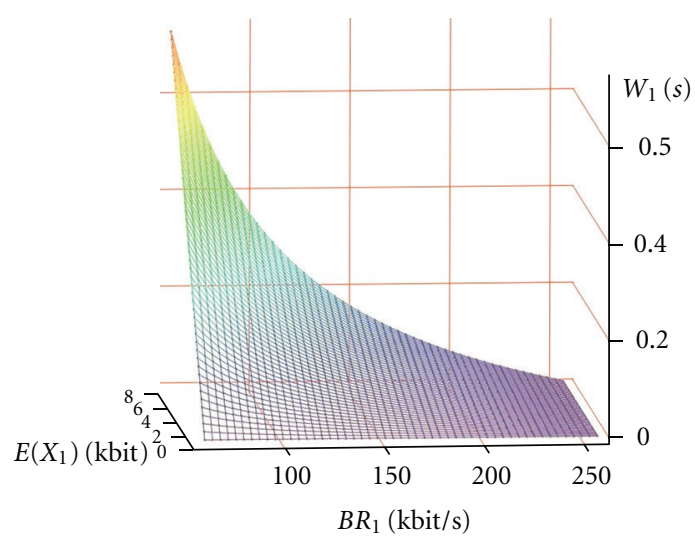

Figure 3: Average waiting time of $i=1$ packets for $\rho_{1 S}=0.93$ and $B R=256 \mathrm{kbps}$.

By substituting of (9) and (11) into the expression (3) we obtain

$$
W_{1}=N_{1}\left[1+\frac{\Delta_{2}}{\Delta_{1}}\right] E\left(T_{1}\right)+\frac{\Delta_{2} E\left(T_{1}\right)}{2 \Delta_{1}} .
$$

The relation between the average number of packets in queue $N_{1}$, the average waiting time of packets $W_{1}$, and processed intensity of entry flow $\lambda_{1 S}$ is expressed by Little's formula:

$$
N_{1}=\lambda_{1 S} \cdot W_{1} .
$$

After substituting Little's formula (13) into the relation (12), we obtain

$$
W_{1}=\frac{\left[\Delta_{2} E\left(T_{1}\right) /\left(2 \cdot \Delta_{1}\right)\right]}{\left[1-\lambda_{1 S} E\left(T_{1}\right) \cdot\left(1+\Delta_{2} / \Delta_{1}\right)\right]} .
$$

After substituting the relation between the average time needed to transfer a packet and the intensity of service $E\left(T_{1}\right)=B R_{1} /\left(B R \cdot \mu_{1}\right)$ to the denominator of the expression (14), introducing the load coefficient of processed traffic $\rho_{1 S}=\lambda_{1 S} / \mu_{1 S}$ and finally after substituting the relation between the average packet size and the average time needed to transfer it $E\left(T_{1}\right)=E\left(X_{1}\right) / B R$ to the numerator of (14), the result will be

$$
W_{1}=\frac{\left[B R_{2} \cdot E\left(X_{1}\right)\right]}{\left[B R_{1} \cdot 2\left(1-\rho_{1 S}\right) \cdot B R\right]} .
$$

From the expression (15) it is obvious that the average time spent in the queue of packets belonging to the first queue $W_{1}$ grows linearly with the average size $E\left(X_{1}\right)$ of these packets. The bigger the packets are being sent, the longer will be their average waiting time $W_{1}$. Further, the average waiting time $W_{1}$ depends on the ratio of sizes of guaranteed proportions $B R_{1}$, and $B R_{2}$ from the overall capacity $B R$. The bigger is the proportion $B R_{1}$, the smaller will be the average waiting time $W_{1}$.

Figure 3 demonstrates the graphic interpretation of the relation (15) for $\rho_{1 S}=0.93$ and $B R=256 \mathrm{kbps}$. Taking 


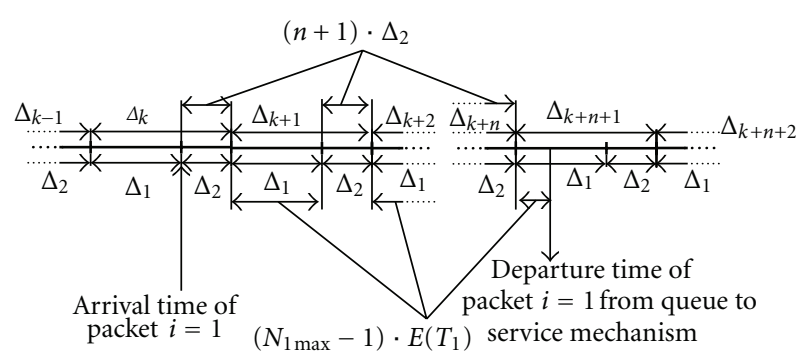

FIgUre 4: Time conditions at the arrival of $i=1$ packet waiting for period $W_{1 \max }$.

Figure 4 into account, it is obvious that the average waiting time $W_{1}$ grows most rapidly in the direction of the growth of average packet size $E\left(X_{1}\right)$ and concurrently to the direction of decrease of the size of portion $B R_{1}$. The smaller the size of the portion $B R_{1}$ is, the steeper will be the increase of the average waiting time $W_{1}$, which depends on the increasing average packet size $E\left(X_{1}\right)$. The bigger the average packet size $E\left(X_{1}\right)$ is, the steeper will be the increase of the average waiting time $W_{1}$ that depends on the decrease of the proportion $B R_{1}$.

The relation for the average waiting time can be generalized using mathematical induction. For the average waiting time of packets of $i$ th queue $W_{i}$, it is valid that

$$
W_{i}=\frac{\left[\left(B R-B R_{i}\right) \cdot E\left(X_{i}\right)\right]}{\left[B R_{i} \cdot 2\left(1-\rho_{i S}\right) \cdot B R\right]} .
$$

The maximum queue length $N_{1 \max }$ can be expressed using the required value of maximum delay of $i=1$ packets [5].

The maximum value of waiting time $W_{1 \max }$ reached by packet $i=1$ will be in the case of entering the system just in the moment when the service of its queue has been finished. Concurrently, just $N_{1 \max }-1$ packets are located before it, so the considered packet is placed in the last position in the queue. For the maximum waiting time $W_{1 \max }$ of packet belonging to the first queue according to Figure 4 , it is valid that

$$
W_{1 \max }=(n+1) \cdot \Delta_{2}+\left(N_{1 \max }-1\right) \cdot E\left(T_{1}\right) .
$$

After deduction for maximum queue length $N_{1 \text { max }}$, we get

$$
N_{1 \max }=\frac{\left[W_{1 \max }+\left(E\left(X_{1}\right) / B R\right) \cdot\left(1+B R_{2} /\left(2 B R_{1}\right)\right)\right]}{\left(E\left(X_{1}\right) / B R\right) \cdot\left(1+B R_{2} / B R_{1}\right)} .
$$

The relation for the maximum queue length can be generalized using mathematical induction. For the maximum queue length of packets of $i$ th queue, it is valid that

$$
N_{i \max }=\frac{\left[W_{i \max }+\left(E\left(X_{i}\right) / B R\right) \cdot\left(1+\left(B R-B R_{i}\right) /\left(2 B R_{i}\right)\right)\right]}{\left(E\left(X_{i}\right) / B R\right) \cdot\left(1+\left(B R-B R_{i}\right) /\left(B R_{i}\right)\right)} .
$$

\section{Implementation of the Mathematical Model and Experimental Results}

The drawn-up mathematical model of a WRR service system was used by configuration of real network architecture emulating a large company network-campus LAN. The created emulating model consists of two segments: LAN 1 (client side segment) and LAN 2 (server side segment). These network parts represent far-flung individual segments of a large company network. The interconnection of individual network segments is created by WAN link emulated by PPP multilink. The data traffic is emulated by multifunctional hardware network emulator. Monitoring of data traffic processing is realized by computers and switches that realize the port mirroring.

Undesirable congestions can especially occur by the transfer of data traffic from high-speed LAN link to lowspeed WAN link [5]. In order to accomplish a reliable voice or video communication, it is necessary to configure suitable QoS tools in the appropriate network devices that guarantee the required transfer quality of multimedia communication [6].

Generated data traffic operations are specified in the emulator event list. Each line in this list presents one required transaction. The emulated users generate load according to event list in the way that progressively require carrying out all events defined in the list. For the needs of emulation, four individual event lists were created. According to the first list the SIP VoIP traffic is generated, according to the second list the RTSP video traffic is generated, according to the third list the SMTP electronic mail traffic is generated, and finally according to the last list the FTP file transfer traffic is generated.

The simplest and in most cases default mechanism of traffic processing is FIFO strategy. It is the elementary packet processing technique that was used as first. For the purpose of detailed statistical analysis of transported data traffic, the additional software application was created. It calculates particular statistical parameters such as the empirical probability distribution of delays of transported packets. In Figure 5 there are demonstrated FIFO/CBWFQ results for voice and video data traffic. The average delay of VoIP RTP packets $W_{1}=652 \mathrm{~ms}$ is unsatisfactory, similarly, average delay of video RTP packets $W_{2}=579 \mathrm{~ms}$ is unsatisfactory. Since processing of interactive packets by FIFO strategy is unsatisfactory from the point of view of quality parameters, it is necessary to implement the more sophisticated mechanism such as CBWFQ. It is needful to create individual operation classes for interactive voice and video packets. Interactive voice RTP packets will belong to the operation class named voice-traffic and video RTP packets will belong to the operation class named video-traffic. For individual operation classes it is necessary to configure the operation parameters that are minimal guaranteed bandwidth and maximal queue length. The setting of these parameters will be realized according to drawn-up mathematical model.

By statistically found-out data traffic parameters (average packet sizes: $E\left(X_{1}\right)=1712$ bit, $E\left(X_{2}\right)=7808$ bit, load coefficients of processed traffic $\left.\rho_{1 S}=0.93, \rho_{2 S}=0.88\right)$, 


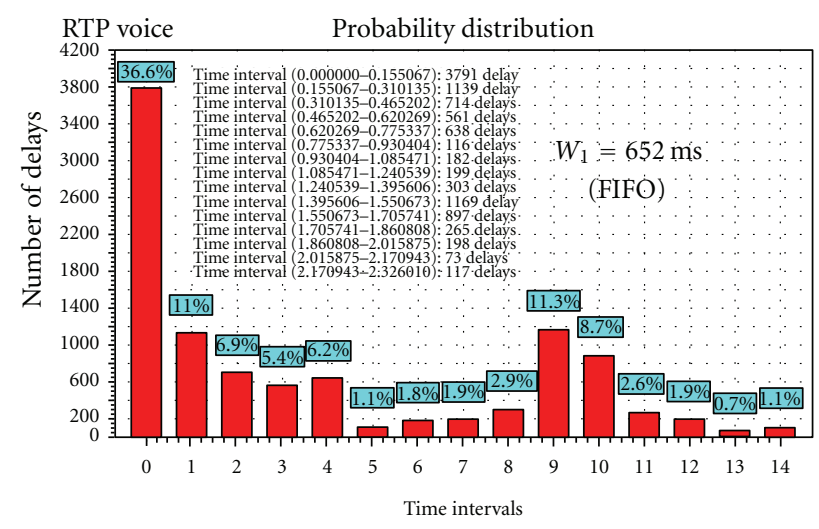

(a)

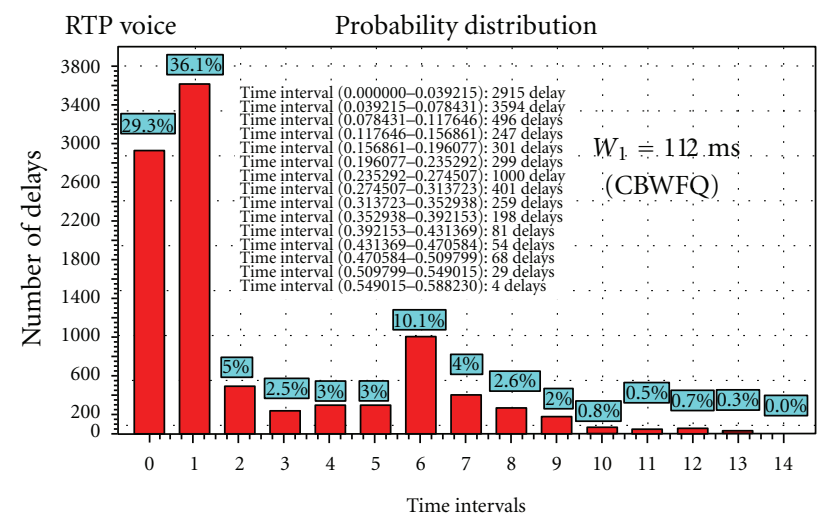

(c)

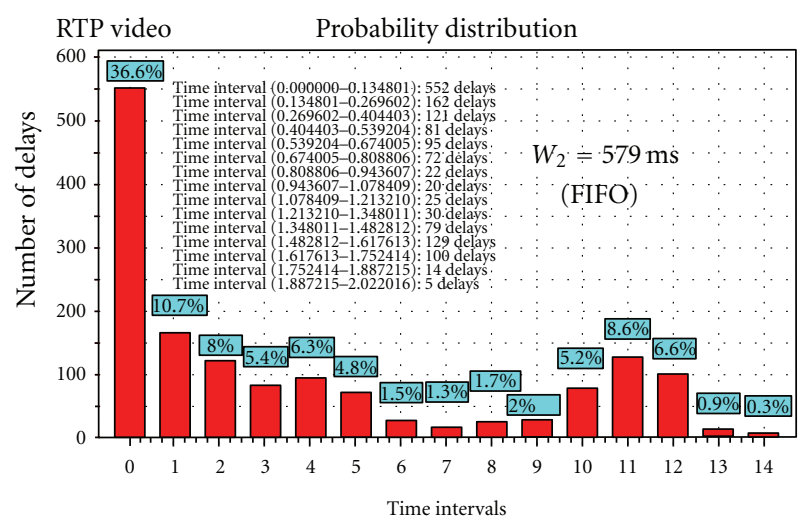

(b)

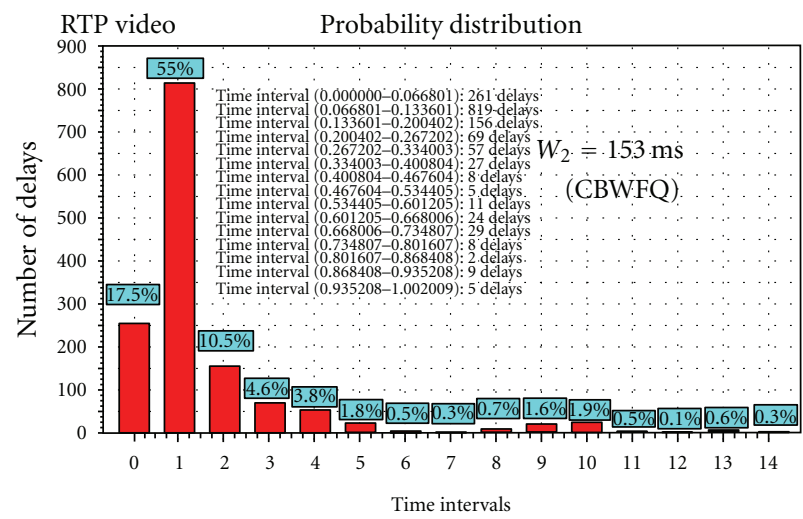

(d)

FIGURE 5: Empirical delay probability distribution for FIFO/CBWFQ strategy.

we can assume that the required average delay of voice RTP packets should be $100 \mathrm{~ms}$ with the peak $700 \mathrm{~ms}$ and required average delay of video RTP packet should be $150 \mathrm{~ms}$ with the peak $8700 \mathrm{~ms}$.

For the size of guaranteed proportion from the total available capacity $B R=256 \mathrm{kbps}$ for the first operation class from the relation (16) we get $\left(B R_{1} / B R\right) \cdot 100 \% \approx 30 \%$ and for the second class we get $\left(B R_{2} / B R\right) \cdot 100 \% \approx 45 \%$.

For the queue length of the first operation class from the relation (19) we get $N_{1 \max } \approx 32$ packets and for the second class we get $N_{2 \max } \approx 128$ packets.

All other packets will be processed without any QoS guarantees as best effort traffic therefore setting operation parameters for this class has no sense $[7,8]$.

In Figure 5 there are demonstrated results for FIFO/CBWFQ strategy. The average delay of VoIP RTP packets compared with FIFO strategy decreased from $652 \mathrm{~ms}$ to $112 \mathrm{~ms}$ and average delay of video RTP packets decreased from $579 \mathrm{~ms}$ to $153 \mathrm{~ms}$. The average delays obtained by emulation are approximately in agreement with required values. The deviations are mostly caused by the finite number of analyzed packets. The considerably lower value of peak delay $W_{2 \max }$ obtained by emulation compared with proposed required value is the result of the fact that the second queue was not even once filled during the emulation. From Figure 5 it is further apparent that approximately $73 \%$ of voice RTP packets have acceptable delay in the range from $0 \mathrm{~ms}$ to $157 \mathrm{~ms}, 25 \%$ of these packets have limit delay value in the range from $157 \mathrm{~ms}$ to $392 \mathrm{~ms}$ and only $2 \%$ of packets have unsatisfactory delay in the range from $392 \mathrm{~ms}$, and more. Similarly, approximately $73 \%$ of video RTP packets have delay in the range from $0 \mathrm{~ms}$ to $134 \mathrm{~ms}, 20 \%$ of these packets have delay in the range from $134 \mathrm{~ms}$ to $401 \mathrm{~ms}$ and only $7 \%$ of packets have delay in the range from $401 \mathrm{~ms}$ and more.

\section{References}

[1] H. M. Chaskar and U. Madhow, "Fair scheduling with tunable latency: a Round Robin approach," in Proceedings of the IEEE Global Telecommunication Conference (GLOBECOM '99), pp. 1328-1333, December 1999.

[2] A. Orda and A. Sprintson, "Precomputation schemes for QoS routing," IEEE/ACM Transactions on Networking, vol. 11, no. 4, pp. 578-591, 2003.

[3] A. Orda, "Routing with end-to-end QoS guarantees in broadband networks," IEEE/ACM Transactions on Networking, vol. 7, no. 3, pp. 365-374, 1999.

[4] B. Adamec, Design of optimization model for data communication management in Canpus LAN, Ph.D. dissertation, Department of Telecommunications and Multimedia, Faculty of Electrical Engineering, University of Zilina, Zilina, Slovakia, 2010. 
[5] X. Liu, K. Ravindran, and D. Loguinov, "A queueing-theoretic foundation of available bandwidth estimation: single-hop analysis," IEEE/ACM Transactions on Networking, vol. 15, no. 4, pp. 918-931, 2007.

[6] V. Paxson, "End-to-end Internet packet dynamics," IEEE/ACM Transactions on Networking, vol. 7, no. 3, pp. 277-292, 1999.

[7] E. Muhammad and S. Stidham, Sample-Path Analysis of Queuing Systems, Kluwer Academic, Boston, Mass, USA, 1999.

[8] C. W. Chan, Performance Analysis of Telecommunications and Local Area Networks, Kluwer Academic, Norwell, Mass, USA, 2000. 

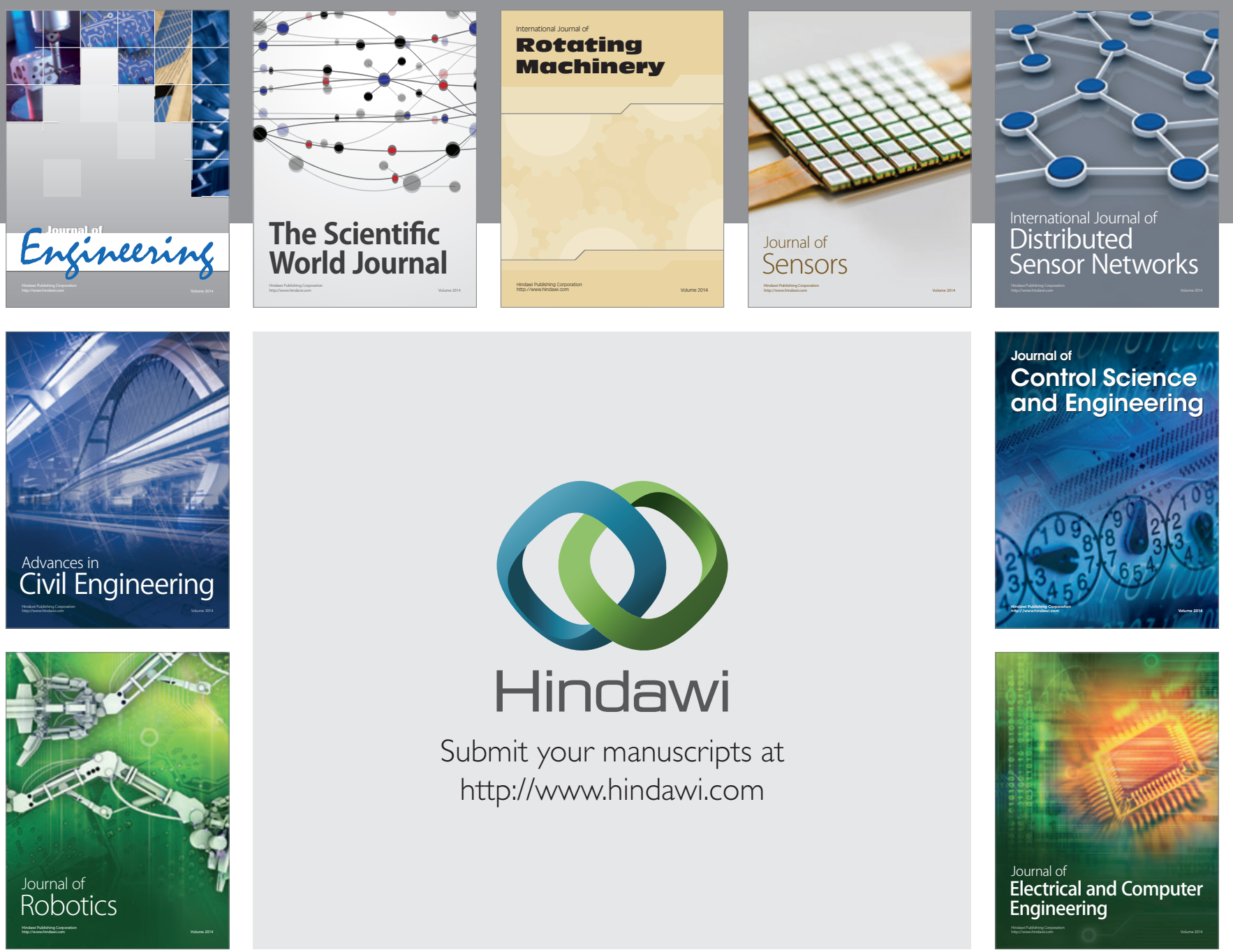

Submit your manuscripts at

http://www.hindawi.com
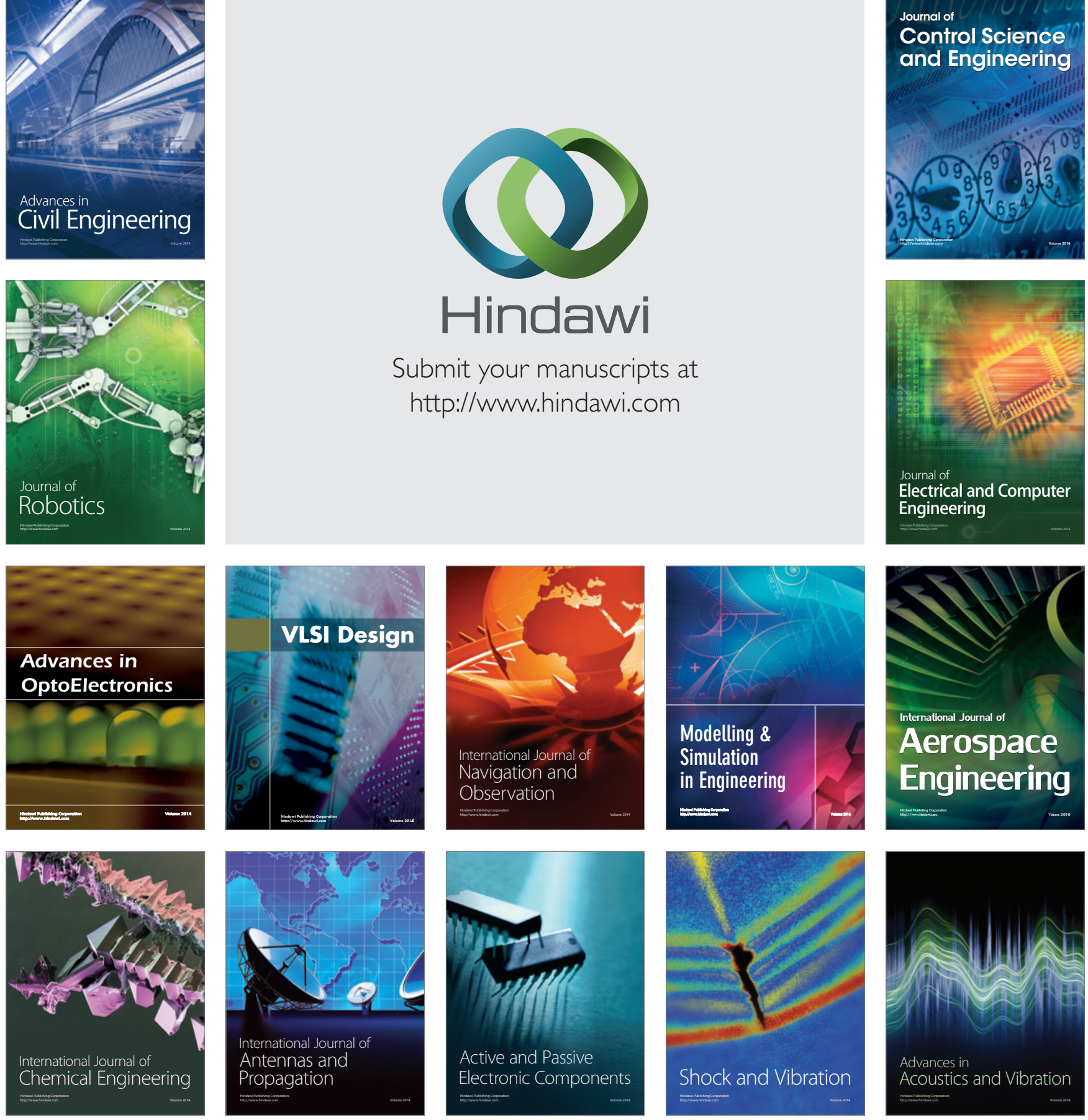